\title{
The Lunar Orbiter Laser Altimeter (LOLA) Laser Transmitter
}

Anthony W. $\mathrm{Yu}^{* a}$, Anne Marie Novo-Gradac ${ }^{\mathrm{a}}$, George B. Shaw ${ }^{\mathrm{a}}$, Glenn Unger ${ }^{\mathrm{b}}$, Luis A. Ramos-Izquierdo ${ }^{c}$, Alan Lukemire ${ }^{\mathrm{d}}$

${ }^{a}$ NASA-GSFC, Laser \& Electro-Optics Branch, MC554, Greenbelt, MD 20771

${ }^{\mathrm{b}}$ NASA-GSFC, Microelectronics and Signal Processing Branch, MC564, Greenbelt, MD 20771

${ }^{c}$ NASA-GSFC, Optics Branch, MC551, Greenbelt, MD 20771

${ }^{\mathrm{d}}$ Space Power Electronics Inc., 108 Audrey Way, Kathleen, GA 31047

\begin{abstract}
We present the final configuration of the space flight laser transmitter as delivered to the LOLA instrument. The laser consists of two oscillators with co-aligned outputs on a single bench, each capable of providing one billion plus shots.
\end{abstract}

Keywords: Lunar Orbiter Laser Altimeter, Laser, Topography, LIDAR, Altimeter, Space laser

\section{INTRODUCTION}

After a series of successful manned missions to the moon by the Apollo program from 1963 to 1972, NASA's plan to return to the moon is initiated by the Lunar Reconnaissance Orbiter (LRO). The LRO is the first mission in NASA's vision for space exploration whose overarching strategy is to conduct investigations and prepare for future human exploration of the Moon, Mars and beyond.' The LRO is scheduled to launch from the Kennedy Space Center in late 2008. The overall mission objective for the LRO is to find and locate landing sites, identify potential resources, characterize the radiation environment and demonstrate new technology. The LRO (Figure 1(a)) baseline mission is nominally 1 Earth year at $30 \times 50 \mathrm{~km}$, polar orbit.

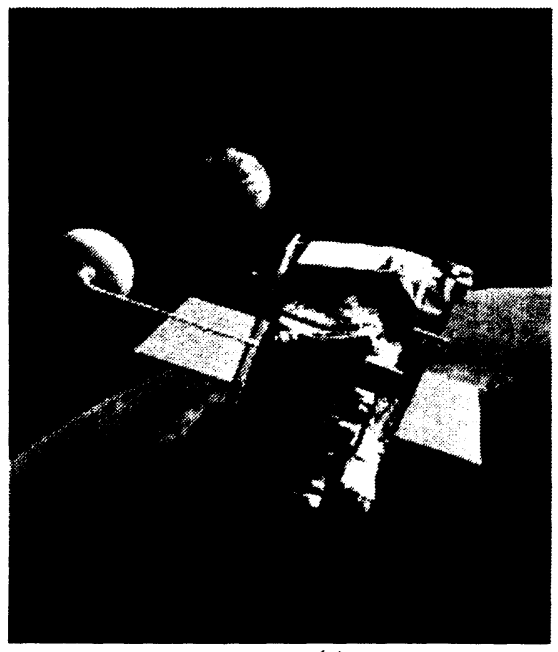

(a)

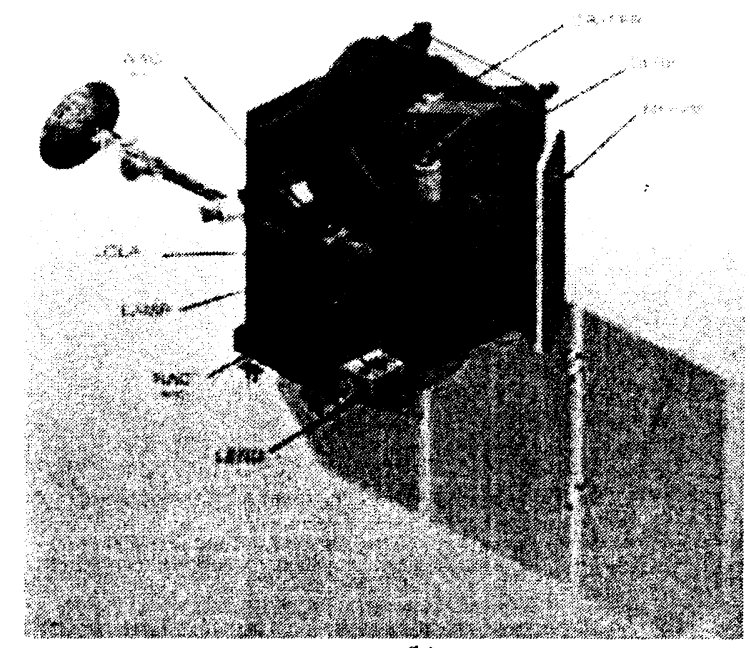

(b)

Figure 1.(a) Rendering of the LRO satellite orbiting the moon; (b) The six scientific instrument on board of LRO, also shown is the Mini-RF on board of the LRO satellite.

The Lunar Orbiter Laser Altimeter (LOLA) instrument is one of seven instruments on the LRO satellite (Figure 1(b)) LOLA will determine the global topography of the lunar surface at high resolution, measure potential landing site slopes and search for polar ices in shadowed regions. The LOLA instrument pulses a single laser through a diffractive optical element $(\mathrm{DOE})^{2}$ to produce five beams that illuminate the lunar surface. For each beam, LOLA measures time of flight (range), pulse spreading (surface roughness), and transmit/return energy (surface reflectance). With its two-dimensional

\footnotetext{
• anthony.w.yu@nasa.gov, phone: 1301286 0206, fax: 1301286 1750; www.gsfc.nasa.gov
} 
spot pattern, LOLA unambiguously determines slopes along and across the orbit track. Analysis of the data at cross-over points from multiple orbital tracks will also provide insight on the gravitational field of the moon and its center of mass.

\section{FLIGHT LASER TRANSMITTER}

The LOLA laser transmitter follows the continued success of $\mathrm{GLAS}^{3}$ and MLA ${ }^{4}$ in using a diode pumped, Cr:Nd:YAG slab with passive q-switch and a cross-Porro resonator configuration. The laser transmitter consists of a beryllium (Be) flight laser bench housing two oscillators (a primary oscillator and a cold spare), an 18X transmit beam expander and a DOE. The two output beams are polarization combined along the beam combining stage as shown in Figure 2(a). The two lasers are designed to operate sequentially during the mission with the secondary laser turning on if the primary laser can no longer provide adequate scientific data products. A picture of the completely assembled LOLA laser on Be bench is shown in Figure 2(a). Figure 2(b) shows the LOLA laser transmitter consisted of the Be laser bench integrated in the Be instrument housing along with the laser electronics assembly (LEA) receive detectors, transmit beam expander and receive telescope. The assembly depicted is undergoing instrument boresight exercise.

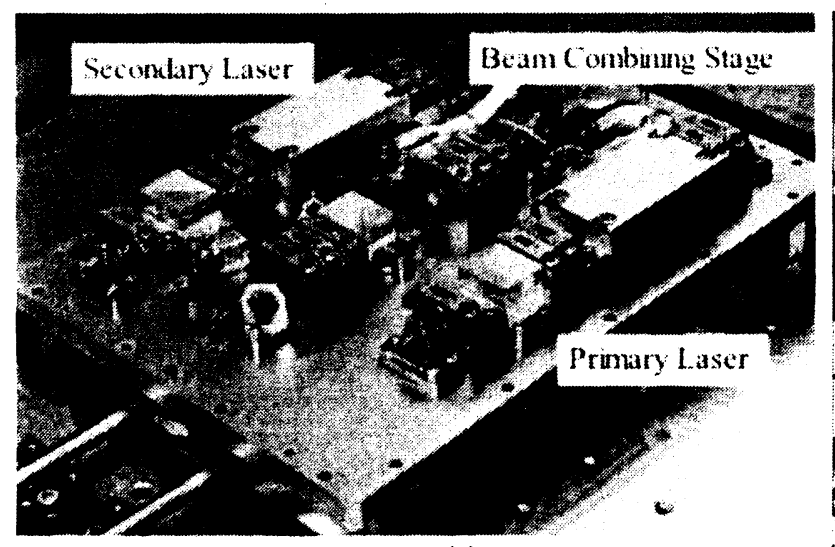

(a)

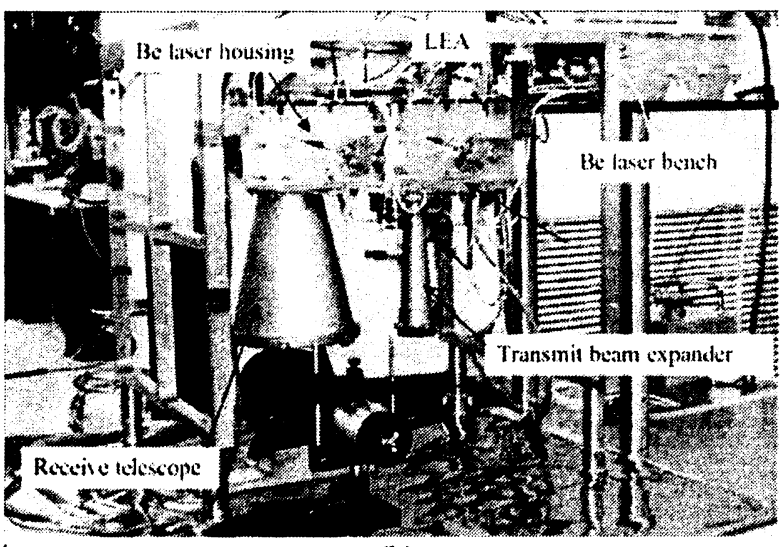

(b)

Figure 2.(a) Picture of the LOLA flight lasers and (b) the LOLA laser transmitter undergoing instrument boresight exercise.

The flight laser transmitter final configuration is different than the engineering model laser transmitter reported earlier. ${ }^{5,6}$ To further improve the reliability of the laser transmitter for a long endurance mission, the internal circulating fluence of the laser oscillator was reduced by lowering the effective output coupling ratio. As reported earlier, 5,6 the output coupling ratio of $50 \%$ was used to allow for $\sim 150 \mu$ s switch out time, $\sim 3 \mathrm{~mJ}$ output energy per pulse with a threshold current of $\sim 60$ A on the pump laser diode array. The total circulating fluence was modeled and found to be $\sim 2.8 \mathrm{~J} / \mathrm{cm}^{2}$ on the high fluence side of the cavity as shown in Figure 3.

The total intracavity fluence was reduced to lower the probability of optical damage during the life of the mission. A factor of two in fluence reduction can be obtained by removing the $0.12 \lambda$ waveplate (WP) on the output side of the cross Porro resonator. The phase retardance provided by the Porro at $45^{\circ}$ relative to the polarization beam splitter (PBS) cube gave an effective output coupling ratio of $\sim 18 \%$. From the system point of view, this decrease in output coupling ratio, or increasing intracavity loss, resulted in an increase of oscillator threshold current and switchout time. At this configuration, the threshold current was $\sim 65 \mathrm{~A}$ with switchout time at $\sim 160 \mu$ s and no change in energy since this laser is designed to operate at constant energy. The operating parameters are well within the system available margin from the instrument. The removal of the $0.12 \lambda$ WP also minimizes the number of optical surfaces within the cavity. 


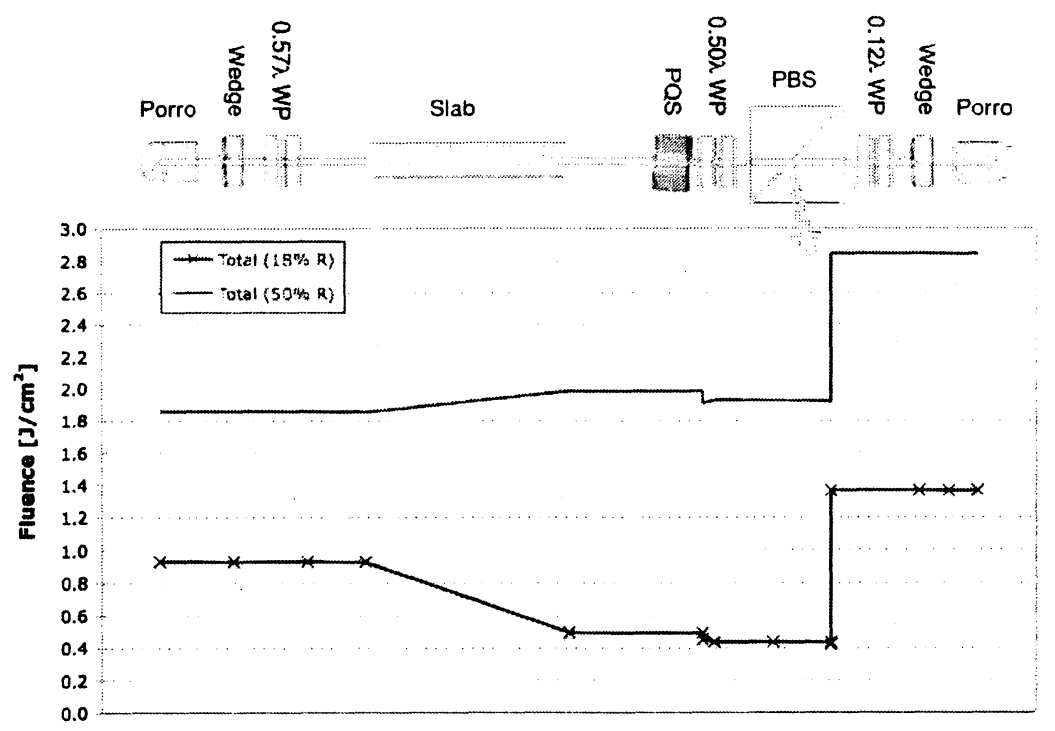

Figure 3. Calculated total intracavity fluence of the LOLA laser cavity. The original configuration of $50 \%$ effective output coupling ratio was achieved by adjusting the $0.12 \lambda$ WP to an appropriate orientation. The new configuration with $18 \%$ effective output coupling ratio was obtained by removing the $0.12 \lambda$ WP from the cavity.

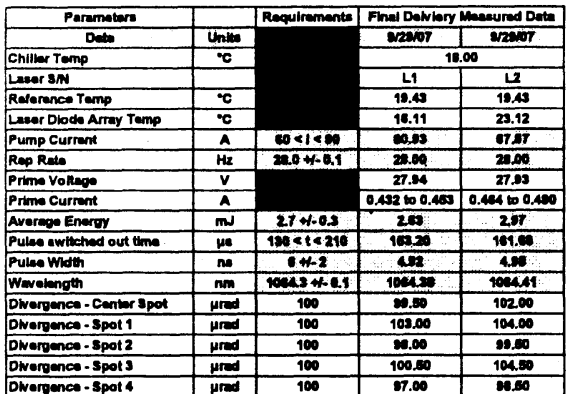

(a)

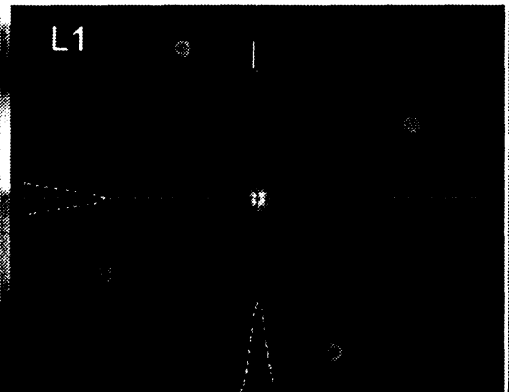

(b)

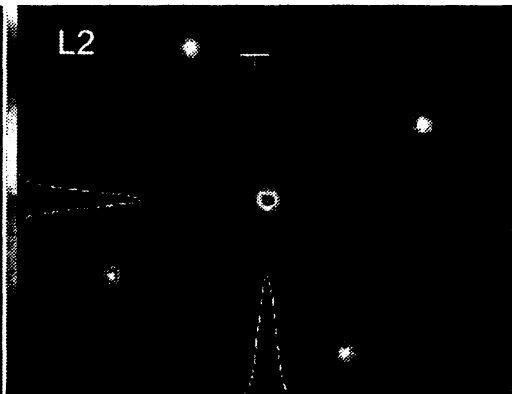

(c)

Figure 4.(a) Summary of the laser pre-delivery performance; (b) Primary (L1) and (c) secondary (L2) laser far field patterns after the beam expander and DOE (the dimmer spot in the upper center is the return from the LOLA instrument alignment reference cube).

The as delivered performance of both primary and secondary lasers is summarized in Figure 4(a). The far field patterns of both lasers after the transmit beam expander and DOE are shown in Figure 4(b). The laser is designed to produce a $2.7 \pm 0.3 \mathrm{~mJ}$ per pulse (prior to transmit beam expander and DOE) at a rate of $28 \mathrm{~Hz}$, pulse width of $6 \pm 2 \mathrm{~ns}$. The far field divergence of each of the five spots is $100 \mu \mathrm{rad}$. At $50 \mathrm{~km}$ orbit, this will produce five- $5 \mathrm{~m}$ diameter spots with centerto-center separation of $25 \mathrm{~m}$ on the lunar surface. The LOLA laser transmitter has successfully undergone vibration testing and temperature cycling in air. Currently the laser transmitter is awaiting instrument level thermal vacuum (TVAC) and vibration tests. We will also be reporting on these environmental test results.

\section{CONCLUSION}

The LOLA instrument is designed to map the lunar surface and provide unprecedented data products in anticipation of future manned flight missions. The laser transmitter design was realized and verified with the engineering model (EM) laser. The LOLA laser transmitter design has heritage dated back to the MOLA laser transmitter launched more than 10 
years ago and incorporates lessons learned from previous missions. The most notable design change in the LOLA laser transmitter from previously flown missions is the use of two vendors laser diode arrays (LDA) with more than $40 \%$ derating. This change will help to enable the LOLA laser to fulfill the mission goal of 1-year continuous operation (or 1 billion shots) in lunar orbit.

\section{ACKNOWLEDGEMENT}

The authors acknowledge the support of the LOLA science team, especially David E. Smith (PI), Maria Zuber (PI), the LOLA instrument team, Glenn Jackson (instrument manager), Ron Zellar (deputy instrument manager), John Cavanaugh (system engineer) and Larry Ramsey (contamination lead).

[1]. http://lunar.gsfc.nasa.gov/index.html.

\section{REFERENCES}

[2]. J.G. Smith, L. Ramos-Izquierdo, A. Stockham, S. Scott, "Diffractive Optics for Moon Topography Mapping," Proc. SPIE, Vol. 6223, 2006.

[3]. R.S. Afzal, A.W. Yu, J.L. Dallas, A. Melak, A. Lukemire, L. Ramos-Izquierdo, W. Mamakos "The Geoscience Laser Altimeter System (GLAS) Laser Transmitter,” IEEE Journ. Of Selected Topics in Quant. Elect., 13, 511-536 (2007).

[4]. D.J. Krebs, A.-M. Novo-Gradac, S.X. Li, S.J. Lindauer, R.S. Afzal, and A.W. Yu, "Compact, passively Q-switched Nd:YAG laser for the MESSENGER mission to Mercury," Appl. Opt. 44, 1715-1718 (2005).

[5]. A.W.Yu, A.-M. Novo-Gradac, G.B. Shaw, J. Cavanaugh, J. Guzek, P. Liiva, L. Nagao, and A. Rosanova, "Thermal vacuum testing of the lunar orbiter laser altimeter engineering model laser," paper 6451-78, presented at Photonics West 2007, San Jose, CA, Jan 2007.

[6]. A.W. Yu, A.M. Novo-Gradac, G.B. Shaw, S.X. Li, M.A. Stephen, D. Krebs, G. Unger, J. Karsh, W. Mamakos, J. Guzek, A. Lukemire, "Design Considerations of the LOLA Laser Transmitter," paper 6451-79 presented at Photonics West 2007, San Jose CA, Jan 2007. 